\title{
ON PRIMES OF THE FORM $u^{2}+5 v^{2}$
}

\section{B. W. BREWER}

1. Introduction. A prime $p$ of the form $20 k+1$ or $20 k+9$ admits of the two integral representations $u^{2}+5 v^{2}$ and $a^{2}+b^{2}$ ( $a$ odd), each representation being essentially unique. Moreover, the only primes other than 5 admitting of the first representation are those of the indicated form. If $p$ is a prime of the form $20 k+1$ or $20 k+9$ and $a \neq 0(\bmod 5)$, the author [1] has expressed $u$ in terms of the sum $\Lambda_{5}=\sum_{x=0}^{p-1} \chi\left(x\left(x^{4}-5 x^{2}+5\right)\right)$, where $\chi(m)$ is the quadratic character of $m$ modulo $p$ and $x\left(x^{4}-5 x^{2}+5\right)$ is the fifth term of the sequence $V_{1}(x)=x, \quad V_{2}(x)=x^{2}-2, \quad V_{n+2}(x)=x V_{n+1}(x)-V_{n}(x) \quad(n=1,2, \cdots)$ (see also A. L. Whiteman, [3], [4]). However, if $a \equiv 0(\bmod 5), \Lambda_{5}=0$. In this paper, we consider the sequence $V_{1}(x, Q)=x, V_{2}(x, Q)=x^{2}$ $-2 Q, V_{n+2}(x, Q)=x V_{n+1}(x, Q)-Q V_{n}(x, Q) \quad(n=1,2, \cdots), Q$ an integer, and study the sum $\Lambda_{5}(Q)=\sum_{\substack{p-1 \\ x=0}}^{p-1}\left(V_{5}(x, Q)\right)$. If $p$ is a prime having one of the above forms, we show in general that $\Lambda_{5}(Q)= \pm 4 u$ when $\chi(Q)=1$ and $a \neq 0(\bmod 5)$ or when $\chi(Q)=-1$ and $a \equiv 0(\bmod 5)$. Specifically, Theorem 2 is concerned with the first case and Theorem 3 with the second case. Theorem 2 reduces to Theorem 4 of [1] when $Q=1$, and refinements of Theorem 3 for certain classes of primes and specific values of $Q$ appear as Corollary 1 and Corollary 2.

Thanks are due the referee for suggesting certain improvements in Theorem 3.

2. Four lemmas. Let $G F\left(p^{m}\right)$ denote the finite field of $p^{m}$ elements ( $p$ a prime). We state Lemma 1 of [1] for completeness.

Lemma 1. If $p$ is an odd prime, $\lambda$ a nonzero element of $G F\left(p^{m}\right)$, and $\lambda$ is of multiplicative period e, then for $s$ a positive integer

$$
\sum_{k=0}^{e-1} \lambda^{k s}= \begin{cases}e & \text { if } s \equiv 0(\bmod e), \\ 0 & \text { if } s \neq \equiv 0(\bmod e) .\end{cases}
$$

The following lemma is a generalization of Lemma 2 of [1].

Lemma 2. Let $p$ be an odd prime and $\lambda$ a generating element of the multiplicative group of $G F\left(p^{2}\right)$. Let $V_{1}(x, Q)=x, V_{2}(x, Q)=x^{2}-2 Q$, $V_{n+2}(x, Q)=x V_{n+1}(x, Q)-Q V_{n}(x, Q)(n=1,2, \cdots)$, where $Q$ is an integer, $\chi(Q)=-1$, and $Q=\lambda^{r(p+1)}(0<r \leqq p-1)$. Let

Presented to the Society, January 26, 1965; received by the editors May 15, 1965 and, in revised form, August 16, 1965. 


$$
\Lambda_{n}(Q)=\sum_{x=0}^{p-1} \chi\left(V_{n}(x, Q)\right), \quad \Omega_{n}(Q)=\sum_{s=0}^{p-2} \chi\left(\lambda^{n s(p+1)}+Q^{n} \lambda^{-n s(p+1)}\right)
$$

and

$$
\Theta_{n}(Q)=\sum_{t=0}^{p} \chi\left(\lambda^{n(t(p-1)+r)}+Q^{n} \lambda^{-n(t(p-1)+r)}\right) .
$$

Then $2 \Lambda_{n}(Q)=\Omega_{n}(Q)+\Theta_{n}(Q)(n=1,2, \cdots)$.

We note that the conclusion of Lemma 2 also follows if $\chi(Q)=1$, but we do not have need for this case.

Proof of Lemma 2. Consider the quadratics $y^{2}-P y+Q$ obtained by letting $P$ run over the set $0,1, \cdots, p-1$, and let $\Delta=P^{2}-4 Q$. Since $\chi(Q)=-1$ and $\sum_{P=0}^{p-1} \chi(\Delta)=-1$, we obtain $(p-1) / 2$ quadratics with $\chi(\Delta)=1$ and $(p+1) / 2$ quadratics with $\chi(\Delta)=-1$. If $\chi(\Delta)$ $=1$, the roots of $y^{2}-P y+Q=0$ in $G F\left(p^{2}\right)$ are of the form $\lambda^{s(p+1)}$ $\lambda^{(r-s)(p+1)}$ for some $s, 0 \leqq s \leqq p-2$. If $\chi(\Delta)=-1$, the roots of $y^{2}-P y$ $+Q=0$ in $G F\left(p^{2}\right)$ are of the form $\lambda^{t(p-1)+r}, \lambda^{(r-t)(p-1)+r}$ for some $t$, $0 \leqq t \leqq p$. Conversely, $\lambda^{s(p+1)}, \lambda^{(r-s)(p+1)}$ are roots of $y^{2}-P y+Q=0$ for some integer $P$ such that $\chi(\Delta)=1$, and $\lambda^{t(p-1)+r}, \lambda^{(r-t)(p-1)+s}$ are roots of $y^{2}-P y+Q=0$ for some integer $P$ such that $\chi(\Delta)=-1$.

Let $H$ denote the set of pairs $\alpha_{s}=\lambda^{s(p+1)}, \alpha_{s}^{\prime}=\lambda^{(r-s)(p+1)}(s=0,1$, $\cdots, p-2)$ and $K$ denote the set of pairs $\beta_{t}=\lambda^{t(p-1)+r}$, and $\beta_{t}^{\prime}$ $=\lambda^{(r-t)(p-1)+r}(t=0,1, \cdots, p)$. Now $\alpha_{i}=\alpha_{j}$ if and only if $i=j$, and $\alpha_{i}=\alpha_{j}^{\prime}$ if and only if $i+j \equiv r(\bmod p-1)$. Likewise, $\beta_{i}=\beta_{j}$ if and only if $i=j$, and since $r$ is odd, $\beta_{i}=\beta_{j}^{\prime}$ if and only if $i+j \equiv r(\bmod p+1)$. Hence there are $(p-1) / 2$ distinct pairs in the set $H$, each pair occurring twice, and $(p+1) / 2$ distinct pairs in the set $K$, each pair occurring twice. Since $\Omega_{n}(Q)=\sum_{s=0}^{p-2} \chi\left(\alpha_{s}^{n}+\alpha_{s}^{\prime \prime}\right)$ and $\Theta_{n}(Q)$ $=\sum_{t=0}^{p} \chi\left(\beta_{t}^{n}+\beta_{t}^{\prime n}\right)$, the lemma follows.

Applying Euler's criterion to $\Omega_{n}(Q)$ and $\Theta_{n}(Q)$ in Lemma 2, we obtain

Lemma 3. Let $\lambda, \Omega_{n}(Q)$ and $\Theta_{n}(Q)$ be defined as in Lemma 2. Then

$$
\Omega_{n}(Q)=\sum_{h=0}^{(p-1) / 2} \sum_{s=0}^{p-2}\left(\begin{array}{c}
(p-1) / 2 \\
h
\end{array}\right) Q^{n h} \lambda^{n s(p+1)(p-4 h-1) / 2}
$$

and

$$
\Theta_{n}(Q)=\sum_{h=0}^{(p-1) / 2} \sum_{t=0}^{p}\left(\begin{array}{c}
(p-1) / 2 \\
h
\end{array}\right) Q^{n h} \lambda^{n(t(p-1)+r)(p-4 h-1) / 2}
$$

in $G F\left(p^{2}\right)$. 
Whiteman has given a proof of the following lemma. Part (1) is proved in [3] and part (2) in [4].

Lemma 4. (1) If $p$ is prime and $p=20 k+1=u^{2}+5 v^{2}=a^{2}+b^{2}$ (a odd), then

$$
\left(\begin{array}{c}
10 k \\
k
\end{array}\right)\left(\begin{array}{c}
10 k \\
3 k
\end{array}\right) \equiv 4 u^{2}(\bmod p)
$$

and

$$
\left(\begin{array}{c}
10 k \\
k
\end{array}\right) \equiv-\left(\begin{array}{c}
10 k \\
3 k
\end{array}\right) \quad \text { or } \quad\left(\begin{array}{c}
10 k \\
k
\end{array}\right) \equiv\left(\begin{array}{c}
10 k \\
3 k
\end{array}\right)(\bmod p)
$$

according as $a \equiv 0(\bmod 5)$ or $a \neq 0(\bmod 5)$.

(2) If $p$ is prime and $p=20 k+9=u^{2}+5 v^{2}=a^{2}+b^{2}$ ( $a$ odd), then

$$
\left(\begin{array}{c}
10 k+4 \\
k
\end{array}\right)\left(\begin{array}{c}
10 k+4 \\
3 k+1
\end{array}\right) \equiv 4 u^{2}(\bmod p)
$$

and

$$
\left(\begin{array}{c}
10 k+4 \\
k
\end{array}\right) \equiv-\left(\begin{array}{c}
10 k+4 \\
3 k+1
\end{array}\right) \text { or }\left(\begin{array}{c}
10 k+4 \\
k
\end{array}\right) \equiv\left(\begin{array}{c}
10 k+4 \\
3 k+1
\end{array}\right)(\bmod p)
$$

according as $a \equiv 0(\bmod 5)$ or $a \neq 0(\bmod 5)$.

3. $\Lambda_{5}(Q)$. We first prove

TheOREM 1. Let $p$ be an odd prime, $\Lambda_{n}(Q)$ be defined as in Lemma 2, and $\chi(Q)= \pm 1$. If $\chi\left(Q^{\prime}\right)=\chi(Q)$ and $Q^{\prime} \equiv m^{2} Q(\bmod p)$, then $\Lambda_{n}\left(Q^{\prime}\right)$ $=\chi(m)^{n} \Lambda_{n}(Q)(n=1,2, \cdots)$.

Proof. Clearly, Theorem 1 will follow if we show that

$$
V_{n}\left(m x, Q^{\prime}\right) \equiv m^{n} V_{n}(x, Q)(\bmod p)
$$

for $n=1,2, \cdots$. We use induction. Now (1) is certainly true for $n=1$ and $n=2$. Assume (1) to be true for all $k<n$. Then

$$
\begin{aligned}
V_{n}\left(m x, Q^{\prime}\right) & \equiv m x V_{n-1}\left(m x, Q^{\prime}\right)-Q^{\prime} V_{n-2}\left(m x, Q^{\prime}\right) \\
& \equiv m x m^{n-1} V_{n-1}(x, Q)-m^{2} Q m^{n-2} V_{n-2}(x, Q) \\
& \equiv m^{n}\left[x V_{n-1}(x, Q)-Q V_{n-2}(x, Q)\right] \equiv m^{n} V_{n}(x, Q)(\bmod p),
\end{aligned}
$$

and Theorem 1 is proved.

Noting that $\Lambda_{5}(Q)=\sum_{\substack{x=0 \\ p-1}} \chi\left(x\left(x^{4}-5 Q x^{2}+5 Q^{2}\right)\right)$, Theorem 1 and Theorem 4 of [1] imply 
Theorem 2. Let $p$ be an odd prime $(p \neq 5), \chi(Q)=1$, and $Q \equiv m^{2}$ $(\bmod p)$. If $p \neq u^{2}+5 v^{2}$, then $p=20 k+r(r=3,7,11,13,17$, or 19) and

$$
\sum_{x=0}^{p-1} \chi\left(x\left(x^{4}-5 Q^{2}+5 Q^{2}\right)\right)=0 .
$$

If $p=u^{2}+5 v^{2}$, then either $p=20 k+1=a^{2}+b^{2}(a \equiv 1(\bmod 4))$, and

$$
\begin{aligned}
\sum_{x=0}^{p-1} \chi\left(x \left(x^{4}-5 Q x^{2}\right.\right. & \left.\left.+5 Q^{2}\right)\right) \\
& =\left\{\begin{array}{l}
0 \quad \text { if } a \equiv 0(\bmod 5), \\
-4 u \chi(m)(u \equiv a(\bmod 5))
\end{array} \text { if } a \neq 0(\bmod 5),\right.
\end{aligned}
$$

or $p=20 k+9=a^{2}+b^{2}(a \equiv 1(\bmod 4))$, and

$$
\begin{aligned}
\sum_{x=0}^{p-1} \chi\left(x \left(x^{4}-5 Q x^{2}+\right.\right. & \left.\left.5 Q^{2}\right)\right) \\
& =\left\{\begin{array}{l}
0 \quad \text { if } a \equiv 0(\bmod 5), \\
4 u \chi(m)(u \equiv a(\bmod 5)) \text { if } a \neq 00(\bmod 5) .
\end{array}\right.
\end{aligned}
$$

To obtain a representation of $u$ in terms of a character sum under the hypothesis that $a \equiv 0(\bmod 5)$, we consider $\Lambda_{5}(Q)$ where $\chi(Q)=-1$. We prove

Theorem 3. Let $p$ be an odd prime $(p \neq 5)$ and $\chi(Q)=-1$. If $p \neq u^{2}$ $+5 v^{2}$, then $p=20 k+r(r=3,7,11,13,17$, or 19), and

$$
\sum_{x=0}^{p-1} \chi\left(x\left(x^{4}-5 Q x^{2}+5 Q^{2}\right)\right)=0 .
$$

If $p=u^{2}+5 v^{2}$, then either $p=20 k+1=a^{2}+b^{2}(a \equiv 1(\bmod 4)$, $\left.b \equiv a Q^{(p-1) / 4}(\bmod p)\right)$, and

$$
\sum_{x=0}^{p-1} \chi\left(x\left(x^{4}-5 Q x^{2}+5 Q^{2}\right)\right)=\left\{\begin{array}{l}
0 \text { if } a \neq 0(\bmod 5), \\
-4 u(u \equiv b(\bmod 5)) \text { if } a \equiv 0(\bmod 5),
\end{array}\right.
$$

or $p=20 k+9=a^{2}+b^{2}\left(a \equiv 1(\bmod 4), b \equiv a Q^{(p-1) / 4}(\bmod p)\right)$, and

$$
\sum_{x=0}^{p-1} \chi\left(x\left(x^{4}-5 Q x^{2}+5 Q^{2}\right)\right)=\left\{\begin{array}{l}
0 \quad \text { if } a \neq \equiv(\bmod 5), \\
4 u(u \equiv b(\bmod 5)) \text { if } a \equiv 0(\bmod 5) .
\end{array}\right.
$$

Proof. That $p=u^{2}+5 v^{2}$ if and only if $p=20 k+1$ or $p=20 k+9$ is well known. We are concerned, therefore, with the evaluation of the sum $\Lambda_{5}(Q)$. If $p=20 k+r(r=3,7,11$, or 19$), \Lambda_{5}(Q)=0$ since $V_{5}(-x, Q)$ $=-V_{\mathfrak{5}}(x, Q)$ and $\chi(-1)=-1$. If $p=20 k+r(r=13$ or 17$)$, we apply Lemma 3 and then Lemma 1 to $\Omega_{5}(Q)$ and $\Theta_{5}(Q)$. We obtain 


$$
\Omega_{5}(Q) \equiv(p-1)\left(\begin{array}{l}
(p-1) / 2 \\
(p-1) / 4
\end{array}\right) Q^{5(p-1) / 4}(\bmod p)
$$

and

$$
\Theta_{5}(Q) \equiv(p+1)\left(\begin{array}{l}
(p-1) / 2 \\
(p-1) / 4
\end{array}\right) Q^{5(p-1) / 4}(\bmod p) .
$$

Hence from Lemma 2 , we have $\Lambda_{5}(Q) \equiv 0(\bmod p)$. Since $\Lambda_{5}(Q)$ is even and numerically less than $p$, this in turn implies that $\Lambda_{5}(Q)=0$.

To obtain the value of $\Lambda_{5}(Q)$ when $p=u^{2}+5 v^{2}$, we again apply Lemma 1 and Lemma 3 to $\Omega_{5}(Q)$ and $\Theta_{5}(Q)$. If $p=20 k+1$, we obtain

$$
\begin{aligned}
\Omega_{5}(Q) \equiv & 2(p-1)\left[\left(\begin{array}{c}
10 k \\
k
\end{array}\right) Q^{5 k}+\left(\begin{array}{c}
10 k \\
3 k
\end{array}\right) Q^{15 k}\right] \\
& +(p-1)\left(\begin{array}{c}
10 k \\
5 k
\end{array}\right) Q^{25 k}(\bmod p)
\end{aligned}
$$

and

$$
\Theta_{5}(Q) \equiv(p+1)\left(\begin{array}{c}
10 k \\
5 k
\end{array}\right) Q^{25 k}(\bmod p) .
$$

If $p=20 k+9$, we obtain

$$
\Omega_{5}(Q) \equiv(p-1)\left(\begin{array}{c}
10 k+4 \\
5 k+2
\end{array}\right) Q^{5(5 k+2)}(\bmod p)
$$

and

$$
\begin{aligned}
\Theta_{5}(Q) \equiv & (p+1)\left(\begin{array}{c}
10 k+4 \\
k
\end{array}\right)\left[Q^{5 k+2}+Q^{9(5 k+2)}\right] \\
& +(p+1)\left(\begin{array}{c}
10 k+4 \\
3 k+1
\end{array}\right)\left[Q^{3(5 k+2)}+Q^{7(5 k+2)}\right] \\
& +(p+1)\left(\begin{array}{c}
10 k+4 \\
5 k+2
\end{array}\right) Q^{5(5 k+2)}(\bmod p) .
\end{aligned}
$$

Since $\chi(Q)=-1, Q^{(p-1) / 4} \equiv i(\bmod p)$, where $i^{2} \equiv-1(\bmod p)$. Moreover,

$$
\left(\begin{array}{l}
(p-1) / 2 \\
(p-1) / 4
\end{array}\right) \equiv 2 a(\bmod p),
$$

where $a \equiv 1(\bmod 4)$ (Gauss). Hence if $p=20 k+1,(2)$ and (3) give 


$$
\Omega_{5}(Q) \equiv-2\left[\left(\begin{array}{c}
10 k \\
k
\end{array}\right)-\left(\begin{array}{c}
10 k \\
3 k
\end{array}\right)\right] i-2 a i(\bmod p)
$$

and

$$
\Theta_{5}(Q) \equiv 2 a i(\bmod p) ;
$$

and if $p=20 k+9,(4)$ and (5) give

$$
\Omega_{5}(Q) \equiv-2 a i(\bmod p)
$$

and

$$
\Theta_{5}(Q) \equiv 2\left[\left(\begin{array}{c}
10 k+4 \\
k
\end{array}\right)-\left(\begin{array}{c}
10 k+4 \\
3 k+1
\end{array}\right)\right] i+2 a i(\bmod p) .
$$

With a suitable choice of the sign of $u$ when $a \equiv 0(\bmod 5)$, Lemma 4 implies that

$$
\left(\begin{array}{c}
10 k \\
k
\end{array}\right)-\left(\begin{array}{c}
10 k \\
3 k
\end{array}\right) \equiv \begin{cases}0(\bmod p) & \text { if } a \neq 0(\bmod 5), \\
-4 u i(\bmod p) & \text { if } a \equiv 0(\bmod 5),\end{cases}
$$

when $p=20 k+1$, and

$$
\left(\begin{array}{c}
10 k+4 \\
k
\end{array}\right)-\left(\begin{array}{c}
10 k+4 \\
3 k+1
\end{array}\right) \equiv \begin{cases}0(\bmod p) & \text { if } a \neq 0(\bmod 5) \\
-4 u i(\bmod p) & \text { if } a \equiv 0(\bmod 5)\end{cases}
$$

when $p=20 k+9$. Thus if $p=20 k+1$,

$$
\Omega_{5}(Q) \equiv \begin{cases}-2 a i(\bmod p) & \text { if } a \neq 0(\bmod 5), \\ -8 u-2 a i(\bmod p) & \text { if } a \equiv 0(\bmod 5),\end{cases}
$$

and $\Theta_{5}(Q) \equiv 2 a i(\bmod p)$; and if $p=20 k+9, \Theta_{5}(Q) \equiv-2 a i(\bmod p)$ and

$$
\Theta_{5}(Q) \equiv \begin{cases}2 a i(\bmod p) & \text { if } a \neq \equiv(\bmod 5) \\ 8 u+2 a i(\bmod p) & \text { if } a \equiv 0(\bmod 5)\end{cases}
$$

Hence from Lemma 2, we have

$$
\Lambda_{5}(Q) \equiv \begin{cases}0(\bmod p) & \text { if } a \neq \equiv 0(\bmod 5) \\ -4 u(\bmod p) & \text { if } a \equiv 0(\bmod 5)\end{cases}
$$

when $p=20 k+1$, and

$$
\Lambda_{5}(Q) \equiv \begin{cases}0(\bmod p) & \text { if } a \neq 0(\bmod 5) \\ 4 u(\bmod p) & \text { if } a \equiv 0(\bmod 5)\end{cases}
$$

when $p=20 k+9$. 
Since $p \geqq 29$ and $|u|<p^{1 / 2}$, it follows that $|4 u|<p$. Then as before, $\Lambda_{5}(Q)$ being even and numerically less than $p$, (9) and (10) imply that

$$
\Lambda_{5}(Q)= \begin{cases}0 & \text { if } a \neq 0(\bmod 5), \\ -4 u & \text { if } a \equiv 0(\bmod 5),\end{cases}
$$

when $p=20 k+1$, and

$$
\Lambda_{5}(Q)= \begin{cases}0 & \text { if } a \neq 0(\bmod 5) \\ 4 u & \text { if } a \equiv 0(\bmod 5)\end{cases}
$$

when $p=20 k+9$.

Now suppose that $a \equiv 0(\bmod 5)$. Since $p=a^{2}+b^{2}(a \equiv 1(\bmod 4))$, the sign of $b$ can be chosen such that $b \equiv a i(\bmod p)$. Since $\Omega_{5}(Q)$ $=\sum_{x=1}^{p-1} \chi\left(x^{5}+Q^{5} x^{-5}\right)=\sum_{x=0}^{p-1} \chi\left(x\left(x^{10}+Q^{5}\right)\right), \Omega_{5}(Q)$ is even. From Lemma 2, we have $2 \Lambda_{n}(Q)=\Omega_{5}(Q)+\Theta_{5}(Q)$, and hence $\Theta_{5}(Q)$ is even. Moreover, since $p \geqq 29$ and $|b|<p^{1 / 2}$, it follows that $|2 b|<p-1$, and then (7) and (8) imply that $\Theta_{5}(Q)=2 b$ when $p=20 k+1$ and $\Omega_{5}(Q)$ $=-2 b$ when $p=20 k+9$. Then from Lemma 2 , we have $-8 u=2 b$ $+\Omega_{5}(Q)$ if $p=20 k+1$, and $8 u=-2 b+\Theta_{5}(Q)$ if $p=20 k+9$. Now it is easily seen that $\Omega_{5}(Q) \equiv 0(\bmod 5)$ if $p=20 k+1$, and $\Theta_{5}(Q) \equiv 0(\bmod 5)$ if $p=20 k+9$. Hence $u \equiv b(\bmod 5)$ when $p=20 k+1$ or $p=20 k+9$ and Theorem 3 is proved.

If $p$ is prime and $p=8 k+5=a^{2}+b^{2}(a \equiv 1(\bmod 4), b / 2 \equiv 1(\bmod 4))$, E. Lehmer [2] has shown that $2^{(p-1) / 4} \equiv b / a(\bmod p)$. If $p$ is prime and $p=12 k+5=a^{2}+b^{2}(a \equiv 1(\bmod 4), b \equiv a(\bmod 3))$, the author [1] has shown that the Jacobsthal sum $\Phi_{2}(-3)=2 b$, and hence $(-3)^{(p-1) / 4} \equiv \Phi_{2}(-3) / \Phi_{2}(1) \equiv-b / a(\bmod p)$. Using these results and Theorem 1, we obtain the following two corollaries to Theorem 3.

Corollary 1. Let $p$ be a prime of the form $40 k+21$ or $40 k+29$, $\chi(Q)=-1$, and $Q \equiv 2 m^{2}(\bmod p)$. If $p=40 k+21$, then $p=u^{2}+5 v^{2}$ $=a^{2}+b^{2}(b$ even, $b / 2 \equiv 1(\bmod 4))$, and

$$
\begin{aligned}
& \sum_{x=0}^{p-1} \chi\left(x\left(x^{4}-5 Q x^{2}+5 Q^{2}\right)\right) \\
& =\left\{\begin{array}{l}
0 \quad \text { if } a \not \equiv 0(\bmod 5), \\
-4 u \chi(m)(u \equiv b(\bmod 5)) \text { if } a \equiv 0(\bmod 5) .
\end{array}\right.
\end{aligned}
$$

If $p=40 k+29$, then $p=u^{2}+5 v^{2}=a^{2}+b^{2}(b$ even, $b / 2 \equiv 1(\bmod 4))$, and 


$$
\begin{aligned}
\sum_{x=0}^{p-1} \chi\left(x \left(x^{4}-5 Q x^{2}+\right.\right. & \left.\left.5 Q^{2}\right)\right) \\
& =\left\{\begin{array}{l}
0 \quad \text { if } a \neq 0(\bmod 5), \\
4 u \chi(m)(u \equiv b(\bmod 5)) \text { if } a \equiv 0(\bmod 5) .
\end{array}\right.
\end{aligned}
$$

Corollary 2. Let $p$ be a prime of the form $60 k+41$, or $60 k+29$, $\chi(Q)=-1$, and $Q \equiv-3 m^{2}(\bmod p)$. If $p=60 k+41$, then $p=u^{2}+5 v^{2}$ $=a^{2}+b^{2}(a \equiv 1(\bmod 4), b \equiv a(\bmod 3))$, and

$$
\begin{aligned}
\sum_{x=0}^{p-1} \chi\left(x \left(x^{4}-5 Q x^{2}+\right.\right. & \left.\left.5 Q^{2}\right)\right) \\
& =\left\{\begin{array}{l}
0 \text { if } a \neq 0(\bmod 5), \\
4 u \chi(m)(u \equiv b(\bmod 5)) \text { if } a \equiv 0(\bmod 5) .
\end{array}\right.
\end{aligned}
$$

If $p=60 k+29$, then $p=u^{2}+5 v^{2}=a^{2}+b^{2}(a \equiv 1(\bmod 4), b \equiv a(\bmod 3))$, and

$$
\begin{aligned}
\sum_{x=0}^{p-1} \chi\left(x \left(x^{4}-5 Q x^{2}\right.\right. & \left.\left.+5 Q^{2}\right)\right) \\
& =\left\{\begin{array}{l}
0 \text { if } a \neq 0(\bmod 5), \\
-4 u \chi(m)(u \equiv b(\bmod 5)) \text { if } a \equiv 0(\bmod 5) .
\end{array}\right.
\end{aligned}
$$

\section{REFERENCES}

1. B. W. Brewer, On certain character sums, Trans. Amer. Math. Soc. 99 (1961), $241-245$.

2. E. Lehmer, On Euler's criterion, J. Austral. Math. Soc. 1 (1959), 64-70.

3. A. L. Whiteman, Theorems on Brewer and Jacobsthal sums. I, Theory of numbers, pp. 44-55, Proc. Sympos. Pure Math. Vol. 8, Amer. Math. Soc., Providence, R. I., 1965.

4. - Theorems on Brewer and Jacobsthal sums. II, Michigan Math. J. 12 (1965), 65-80.

Texas A \& M UnIVERsity 